\title{
Flexibilität und Anpassungsfähigkeit von Beschäftigten mit dualer Berufsausbildung
}

\author{
Alexandra Fedorets · Alexandra Spitz-Oener
}

Angenommen: 22. März 2011 / Online publiziert: 12. April 2011

(C) Institut für Arbeitsmarkt- und Berufsforschung 2011

Zusammenfassung Dieser Beitrag liefert eine Übersicht über zwei Studien, in denen die Mobilität von Erwerbstätigen untersucht wird, die ihre Ausbildung im Rahmen des dualen Ausbildungssystems gemacht haben. Die theoretische Grundlage beider Studien bildet der Humankapitalund Suchtheorieansatz unter der Annahme variierender Berufsinhalte über die Zeit. Mit Hilfe von Regressionsanalysen wird gezeigt, dass Berufsmobilität positiv mit dem individuellen Lohn korreliert ist. Dabei hängt der Korrelationsgrad von der inhaltlichen Ähnlichkeit des Ausbildungs- und Erwerbsberufs und der Freiwilligkeit der Entscheidung eines Berufswechsels ab. Die Ähnlichkeit des Ausbildungs- und Erwerbsberufs wird anhand des tätigkeitsbasierten Ansatzes gemessen.

Die Auswertung der Berufswechsel in West- und Ostdeutschland nach der Wiedervereinigung 1990 führt zu einer wichtigen Erkenntnis: die meisten Berufswechsel in Ostdeutschland nach 1990 brachten hohe Lohneinbußen mit sich. Dieser negative Effekt der Anpassung der Beschäftigungsstruktur wirkt langfristig nach, da er auch nach ca. 10 Jahren noch feststellbar ist.

Insgesamt deuten die empirischen Ergebnisse darauf hin, dass das während der Ausbildung akkumulierte Humankapital zwischen unterschiedlichen Berufen transferierbar ist.

\section{Flexibility and adaptability of the employees with a dual vocational training degree}

Abstract We examine the occupational and wage mobility of employees with a vocational training degree obtained in

M.Sc. A. Fedorets ( $\varangle)$ · Prof. Dr. A. Spitz-Oener Institut für Angewandte Mikroökonomik

Wirtschaftswissenschaftliche Fakultät, Humboldt-Universität zu Berlin, Spandauer Straße 1, 10178 Berlin, Deutschland e-mail: alexandra.fedorets@staff.hu-berlin.de the German dual system of apprenticeship training. The conceptual framework is based on the human capital theory and the search theory under the assumption of variation in occupational contents over time. The regression analysis shows that occupational changes are positively correlated with individual wages. It provides evidence that the degree of correlation depends on the similarity between the content of occupation in which the individual was trained and the kind of occupation in which the individual was employed at the time of the survey. Further, it shows that the voluntariness of occupational change also contributes to the degree of correlation. We measure the similarity of occupational contents with the task-based approach.

In contrast to the results for West Germany, occupational changes in East Germany after reunification are associated with wage penalties. This negative wage effect is persistent and can be observed even 10 years after the occupational change.

The general results suggest that human capital which was accumulated during apprenticeship training, is transferable across different occupations.

\section{Einleitung}

Das deutsche System der dualen Ausbildung, über das ca. $70 \%$ der jungen Erwachsenen den Berufseinstieg finden (Winkelmann 1996a), ist nicht nur national von großer Bedeutung. Mehrere Studien belegen das rege Interesse internationaler Politiker und Wissenschaftler an dieser Ausbildungsinstitution. Die Wurzeln des Dualen Ausbildungssystems reichen bis in das Mittelalter zurück und sind im Lauf der Jahrhunderte mit wirtschaftspolitischen Institutionen Deutschlands zusammengewachsen. Vor diesem Hintergrund stellt sich die Frage, inwieweit das Duale Ausbil- 
dungssystem den derzeitigen Anforderungen der Arbeitsmärkte gerecht werden kann.

Technologischer Wandel ruft neue Verhältnisse zwischen Kapital, Arbeit und Qualifikation hervor. Aber sind die Beschäftigten mit einer Ausbildung durch das duale Ausbildungssystem in der Lage, sich an die sich stetig ändernden Anforderungen am Arbeitsplatz anzupassen? Möglich wäre es, dass die Absolventen aufgrund einer zu eng gefassten Ausbildung an Hürden scheitern, die durch ein höheres Maß an Flexibilität zu überwinden wären. Mit dem Ziel, diese bildungs- und arbeitsmarktpolitisch höchst relevante Fragestellung empirisch zu erhellen, wird in diesem Beitrag die berufliche Mobilität und daraus resultierende Lohnmobilität ehemaliger Absolventen des dualen Ausbildungssystems untersucht. Die Rolle des Berufswechsels soll mit Hilfe des tätigkeitsbasierten Ansatzes, der Arbeitsinhalte in Tätigkeiten klassifiziert, beleuchtet werden. Als Erfolgsmaß der beruflichen Anpassung wird die Entwicklung des individuellen Lohns verwendet.

Die Analyse gibt Aufschluss über drei Fragen, die im Zusammenhang mit der dualen Berufsausbildung intensiv diskutiert werden:

(1) Befähigt die Ausbildung im Rahmen des dualen Ausbildungssystems Beschäftigte dazu, mit den durch technologischen Wandel veränderten Anforderungen am Arbeitsplatz mitzuhalten?

(2) Wie ist in diesem Zusammenhang der Berufswechsel zu bewerten? Positiv, als eine höhere Stufe auf der individuellen Karriereleiter, oder negativ, als Verlust von Humankapital, d.h. als Hinweis darauf, dass die Beschäftigten den veränderten Anforderungen ihres Berufs nicht gewachsen waren?

(3) Welche Konsequenzen ergeben sich aus den Ergebnissen für das System der dualen Berufsausbildung? Wo sollten Reformbemühungen ansetzen?

\section{Theoretische Grundlagen}

Das Potenzial eines Beschäftigten zur Anpassung seiner Qualifikation entfaltet sich über mehrere Dimensionen. Die geographische Mobilität steht in starkem Zusammenhang mit der regionalen Arbeitsmarktstruktur; sie wird im empirischen Teil des Beitrags nur indirekt berücksichtigt. Die berufliche Mobilität beschäftigt sich mit der Nutzung des Humankapitals beim ersten Einstieg in das Arbeitsleben sowie mit der Verwertbarkeit des Humankapitals nach einer Periode der Arbeitslosigkeit oder nach einem Berufswechsel. Dieser Berufswechsel und die anschließende Entlohnung eines Beschäftigten stehen im Fokus dieses Beitrags.

Berufswechsel sind aus einer modernen Wirtschaft nicht wegzudenken. Das moderne Wirtschaftssystem und der technologische Fortschritt sorgen nicht nur dafür, dass alte
Berufe an Bedeutung verlieren, verschwinden und neue Berufsbilder entstehen, sondern auch dafür, dass sich die Tätigkeitsschwerpunkte einzelner Berufe inhaltlich verschieben. Eine detaillierte Erfassung dieses Aspekts der Arbeitsmarktentwicklung ist möglich, wenn die Inhalte einzelner Berufe direkt berücksichtigt werden können. Aus diesem Grund wird die Rolle des Berufswechsels mit Hilfe des tätigkeitsbasierten Ansatzes analysiert. Dieser definiert den Beruf als eine Zusammensetzung von Tätigkeiten, die teilweise berufsübergreifend eingesetzt werden können.

Theoretische Überlegungen legen drei Aspekte nahe, anhand derer die Flexibilität von Beschäftigten mit einer dualen Ausbildung nach einem Berufswechsel gemessen werden kann:

(1) Verwertung des akkumulierten Humankapitalstocks und seiner Transferierbarkeit zwischen unterschiedlichen Berufen im Fall eines Berufswechsels. In diesem Zusammenhang wird auch von berufsspezifischem $\mathrm{Hu}-$ mankapital gesprochen.

(2) Optimalität des Matches zwischen dem Arbeitnehmer und seiner Karriere, d.h. der Grad, zu dem sich die potentielle Produktivität des Arbeitnehmers in einem bestimmten Beruf entfalten kann (analog zu einem Match zwischen dem Arbeitnehmer und seinem Arbeitsplatz, vgl. Franz 2006). Die Abbildung der Steigerung bzw. Senkung des Wertes eines Arbeitnehmer-Karriere Matches nach einem Berufswechsel erfolgt in der empirischen Literatur durch Verwendung einer binären Variablen, die den Wert eins annimmt, wenn ein Berufswechsel stattgefunden hat und ansonsten den Wert null annimmt. Auf diese Weise wird der direkte Einfluss eines Berufswechsels auf den Lohn gemessen.

(3) Anpassung der Qualifikationen während der Berufsausübung, d.h. die durchgehende Anpassung an sich stetig ändernde Berufsinhalte.

Im Gegensatz zu den ersten beiden Merkmalen für die in der Theorie statische Berufsinhalte angenommen werden, konzentriert sich das dritte Merkmal auf den dynamischen Charakter einzelner Berufe. Obwohl in der Literatur bereits erwähnt, wurde dieses Merkmal noch nicht in empirischen Studien über Berufsmobilität berücksichtigt.

Diese drei Merkmale bilden die Grundlage für die empirischen Analysen, die im Folgenden vorgestellt werden. Zunächst folgt ein Überblick der einschlägigen Literatur. Danach werden die Ergebnisse eigener empirischer Studien vorgestellt.

\section{Literaturübersicht}

Im Zentrum dieses Abschnitts stehen vor allem die Literatur über das deutsche duale Ausbildungssystem als institutionelles Phänomen, Studien über Akkumulation und Transferierbarkeit des Humankapitals, sowie die Suchtheorie und 
empirische Anwendungen des tätigkeitsbasierten Ansatzes. Die umfangreiche Literatur über das deutsche System der dualen Ausbildung konzentriert sich hauptsächlich auf die institutionellen Besonderheiten des nationalen Arbeitsmarktes, sowie auf internationale Vergleiche der institutionellen Hintergründe, die das duale Ausbildungssystem in Deutschland ermöglichen (s. Studien von Harhoff und Kane 1993, 1997; Sharpe und Gibson 2005). Eine detaillierte Beschreibung des Systems liefern z.B. Münch (1995) und Franz und Soskice (1995).

Die Studien über Humankapitalakkumulation sind zugleich mit einer Diskussion über die Rolle der Wirtschaftsakteure verschränkt, die sowohl durch direkte als auch indirekte Investitionen zur Entstehung des Ausbildungssystems beitragen (s. Streek et al. 1987). Nach der Humankapitaltheorie werden Firmen zwar nicht in die Entstehung von allgemeinem Humankapital investieren, weil es sehr leicht transferierbar ist. Die Investitionen in spezifisches Humankapital hingegen sollen nach der Theorie sowohl vom Arbeitgeber, als auch vom Arbeitnehmer getätigt werden. Harhoff und Kane (1993) zeigen, dass der regulatorische Druck $\mathrm{zu}$ einer Standardisierung von Berufen führt und sich das in dem dualen Ausbildungssystem entstandene Humankapital somit als allgemein klassifizieren lässt. In einer Vergleichstudie zeigen auch Korpi und Mertens (2003), dass die Akkumulation des allgemeinen Humankapitals den Ausbildungsinhalt dominiert. Franz und Soskice (1995) und Acemoglu und Pischke (1998) entwickeln anhand der theoretischen Konzepte und empirischen Befunde eine Erklärung, warum gewinnmaximierende Firmen in die Akkumulation von allgemeinem Humankapital der Arbeitnehmer investieren. In ihrer späteren Studie zeigen Acemoglu und Pischke (1999), dass die Kompression der Lohnstruktur in Deutschland die „technologisch“ allgemeinen Qualifikationen in de facto spezifische Qualifikationen umwandelt, was auch die Humankapitalinvestitionen seitens der Arbeitgeber erklärt. Eine weitere Analyse der vorhandenen Kostenstruktur des dualen Ausbildungssystems in Deutschland liefern Harhoff und Kane (1997), die zu dem Schluss kommen, dass die Anreiz- und somit die Investitionsstruktur im dualen Ausbildungssystem tief mit der institutionellen Besonderheit des deutschen Arbeitsmarktes verwurzelt ist, was die Implementierung des System in einem anderen Land, oder einem institutionellen Kontext, unmöglich macht.

Für diesen Beitrag besonders relevant sind Studien, die den Zusammenhang zwischen Arbeitsmobilität der Beschäftigten und ihren Löhnen untersuchen. Eine der ersten Systematisierungen des Phänomens der Arbeitsmobilität wurde von Parnes (1954) erstellt, der zwischen einfachen und komplexen Arbeitsplatzwechseln unterscheidet. Bei einem einfachen Arbeitsplatzwechsel wird nur der Arbeitgeber gewechselt, wohingegen sich der komplexe Wechsel durch Änderungen der Arbeitsinhalte auszeichnet. In Bezug auf die Suchtheorie bedeutet dies, dass man zwischen
Arbeitnehmer-Arbeitgeber und Arbeitnehmer-Karriere Matches unterscheiden sollte. Mit anderen Worten, die Produktivität des individuellen Humankapitals wird gleichzeitig durch die Zugehörigkeit zu dem arbeitgebenden Unternehmen und dem Beruf der Beschäftigung bestimmt. Ein Modell, das die Komplexität des Jobwechsels einbezieht, wurde von Neal (1999) entwickelt. Es impliziert, dass die einfachen Arbeitsplatzwechsel überwiegend von älteren Arbeitnehmern getätigt werden. Die komplexen Berufswechsel finden dem Modell nach häufiger am Anfang einer Karriere statt, u.a. weil junge Arbeitnehmer durch ihre relativ kurze Beschäftigungsdauer weniger Humankapital in einem Beruf gesammelt haben und insofern weniger Kosten bei einem Berufswechsel und eventuell damit verbundener Arbeitslosigkeit tragen. Auf ähnlichen Argumenten basiert auch die Theorie des so genannten „Job Shoppings“ zu Beginn des Arbeitslebens (vgl. Johnson 1978 und Topel und Ward 1992). Die Theorie des „Job Shoppings“ beschreibt das Phänomen, dass Arbeitnehmer dazu tendieren, zu Beginn ihres Berufslebens mehrere Arbeitsplätze auszuprobieren, bis sie einen am besten für sich geeigneten Arbeitgeber finden.

Unterschiedliche Erklärungen und Folgen eines Arbeitsplatzwechsels können in Studien von Hofbauer und Nagel (1987), Herget et al. (1985), Winkelmann (1996a, 1996b), Acemoglu und Pischke (1998), Franz und Zimmermann (1999), Von Wachter und Bender (2006) gefunden werden. Die folgenden Studien haben jedoch die komplexere Form der Arbeitsmobilität zum Gegenstand, also Berufswechsel mit Änderung der Arbeitsinhalte. Die Wahrscheinlichkeit eines Berufswechsels nach einer Berufsausbildung wird u.a. von Hofbauer und Nagel (1987) und Franz und Zimmermann (1999) untersucht. Die Autoren analysieren Berufsgruppen, die ein Überangebot bzw. eine Unterversorgung an Auszubildenden aufweisen, und kommen zu dem Schluss, dass das Überangebot an Auszubildenden in Kombination mit niedriger Übertragbarkeit der Qualifikationen eines Berufes zu einer höheren Wahrscheinlichkeit der Arbeitslosigkeit führt. Darüber hinaus unterstreicht Werwatz (1997) interessante Messunterschiede. Die Studien, die auf Eigenauskunft der Befragten basieren, weisen ein tendenziell niedrigeres Niveau der Berufsmobilität auf als Studien, die einen Berufswechsel anhand der Berufskodierungen dokumentieren. Eine mögliche Erklärung dafür ist, dass die Daten, die auf einer Selbsteinschätzung basieren, auch die Berufsinhalte berücksichtigen. D.h. dass ein Befragter nur dann von einem Berufswechsel berichtet, wenn sich die Tätigkeiten, die er am Arbeitsplatz ausübt, signifikant ändern.

Mit Blick auf Lohnveränderungen wird der Effekt eines Berufswechsels generell positiv eingeschätzt (siehe z.B. die Studie von Werwatz 1997). Fitzenberger und Spitz (2004) benutzen die BIBB/IAB-Erwerbstätigenbefragung, um die Einflussfaktoren des Berufswechsels, u.a. den entsprechenden Lohneffekt, zu untersuchen. Sie finden heraus, dass 
ein Berufswechsel die Karriereaussichten steigert und dass das deutsche duale Ausbildungssystem eine Akkumulation von transferierbarem Humankapital fördert. In diesem $\mathrm{Zu}$ sammenhang zeigt die Studie von Fitzenberger und Kunze (2005), dass Lohnzuwächse durch strategisch eingesetzte Berufswechsel häufiger bei Männern als bei Frauen zu beobachten sind.

Die empirische Literatur, die Hintergründe von Berufswechsel erfasst, ist sehr knapp. Empirische Studien über die Arbeitsinhalte waren mangels geeigneter Daten lange Zeit nicht möglich. Eine der ersten prominenten Studien, in der Berufsinhalte als eine Kombination von Tätigkeiten begriffen werden, stammt von Autor et al. (2003). Mit diesem tätigkeitsbasierten Ansatz erklären die Autoren, wie computerbasierte Technologien als Substitute oder Komplemente zu einzelnen Tätigkeiten (Routine- und Nicht-RoutineTätigkeiten) auftreten und wie dadurch Nachfrageverschiebungen für unterschiedliche Qualifikationsgruppen entstehen können. Die meisten Studien, die an den tätigkeitsbasierten Ansatz anknüpfen, verwenden die BIBB/IABErwerbstätigenbefragung, die eine verlässliche Datenquelle über Ausbildungen und Berufsinhalte auf Mikroebene darstellt. Die hohe Qualität dieser Daten ermöglicht es SpitzOener (2006), den tätigkeitsbasierten Ansatz für den deutschen Kontext weiter zu entwickeln und die steigende Nachfrage nach Bildung empirisch zu belegen. Eine direkte Anwendung des tätigkeitsbasierten Ansatzes wird von Gathmann und Schoenberg (2009) durchgeführt. In ihrer Studie entwickeln sie ein multidimensionales Maß für die Unterschiedlichkeit zwischen verschiedenen Berufsinhalten und führen das Konzept des tätigkeitsspezifischen Humankapitals ein. Anhand dieses Maßes wird gezeigt, dass die meisten Berufswechsel zwischen Berufen stattfinden, deren Inhalte ähnlich sind. Außerdem ermitteln sie, dass etwa $20 \%$ des Lohnwachstums eines Individuums auf die Akkumulation des tätigkeitsspezifischen Humankapitals zurückgeführt werden kann.

Die Problematik der sich ständig ändernden Berufsinhalte wird von manchen Autoren erwähnt (z.B. Parnes 1954; Franz und Zimmermann 1999; Ehrenberg und Smith 2006; Spitz-Oener 2006), allerdings nicht weiter in ihren Untersuchungen berücksichtigt.

In der vorliegenden empirischen Analyse wird auf eine modifizierte Version des Ansatzes von Gathmann und Schoenberg (2009) zurückgegriffen, die zusätzlich sich über die Zeit variierende Berufsinhalte zulässt.

\section{Zusammenfassung eigener Studien}

Im Folgenden wird eine zusammenfassende Darstellung und Diskussion von zwei Aufsätzen Fedorets (2011a, 2011b) vorgestellt, die im DFG-Projekt „Flexibilität und Anpassungsfähigkeit Beschäftigter mit Ausbildung im Rahmen der dualen Berufsausbildung" entstanden sind.

\subsection{Daten}

Die Analyse wird auf Grundlage der Umfrage „Erwerb und Verwertung beruflicher Qualifikation von Erwerbstätigen“ durchgeführt. Diese repräsentative Erwerbstätigenbefragung wurde gemeinsam vom Bundesinstitut für Berufsbildung (BiBB) und vom Institut für Arbeitsmarkt- und Berufsforschung (IAB) erhoben. Die Stichproben der vorliegenden Studien beschränken sich auf erwerbstätige Männer mit Berufsausbildung als höchste Bildungsstufe. Der wichtigste Vorteil des Datensatzes liegt darin, dass er Informationen über die Berufsinhalte, d.h. die konkreten Tätigkeiten der Beschäftigten am Arbeitsplatz enthält. Diese Informationen bilden die Grundlage für verschiedene Tätigkeitsmaße und werden oft in der empirischen Arbeit benutzt; sie machen den Datensatz international einmalig. Aufschlussreich ist der Datensatz auch deswegen, weil er retrospektive Informationen über den Verlauf des Arbeitslebens der Befragten enthält. Beispielsweise gibt der Datensatz Auskunft darüber, in welchem Beruf er zum Zeitpunkt der Befragung tätig ist, seit wann er bei seinem jetzigen Arbeitgeber beschäftigt ist, in welchem Beruf er seine Ausbildung gemacht hat und in welchem Jahr die Ausbildung absolviert wurde. Anhand dieser Informationen kann festgestellt werden, ob der Befragte in seinem Ausbildungsberuf tätig ist, oder ob er im Laufe seiner Karriere einen Berufswechsel vorgenommen hat. In der Kombination mit den im Beruf ausgeübten Tätigkeiten lassen sich auch die Entwicklungen in den Berufsinhalten in unterschiedlichen Jahren feststellen. Darüber hinaus enthält der Datensatz Informationen sowohl über die Region des derzeitigen Wohnsitzes (West- oder Ostdeutschland), als auch die Herkunftsregion. Dies ermöglicht zuverlässige Aussagen darüber, ob der Befragte seine Karriere nur in West- bzw. Ostdeutschland, oder überregional verfolgt hat.

\subsection{Empirische Ergebnisse}

Der erste Teil der empirischen Analyse beschäftigt sich mit der Gegenüberstellung folgender drei Komponenten:

- Transferierbarkeit des Humankapitals;

- Wert des Arbeitnehmer-Karriere Matches;

- Wandel der Arbeitsanforderungen über die Zeit.

Die erste und die dritte Komponente werden auf Basis des tätigkeitsbasierten Ansatzes nach Gathmann und Schoenberg (2009) berechnet. Dabei erlauben die im Datensatz enthaltenen Informationen einen 13-Dimensionalen Vektor mit ausgeübten Tätigkeiten zu bilden. Mit anderen 
Worten kann jeder Beruf als ein Punkt in einem mehrdimensionalen Tätigkeitenraum dargestellt werden, wobei die genaue Lage dieser Punkte von den jeweiligen Befragungsjahren abhängt. Diese Darstellung ermöglicht nicht nur die Berechnung der Entfernung zwischen den Tätigkeitsinhalten zwischen zwei Berufen, sondern auch die Erfassung der Entwicklungen, denen die einzelnen Berufe im Laufe der Zeit unterliegen. Für die empirische Umsetzung bedeutet das, dass drei unterschiedliche Variablen in der Regression benutzt werden. Die erste Variable steht für die tätigkeitsbasierten Entfernungen zwischen dem Ausbildungs- und Erwerbsberuf im Fall eines Berufswechsels. Die zweite Variable beinhaltet die Entfernung zwischen den Inhalten der Ausbildungs- und Erwerbsberufs, wenn kein formaler Berufswechsel stattfindet. Diese Variable umfasst die inhaltlichen Änderungen, die der Beschäftigte in seinem Beruf erfährt, aber auch das von ihm im Beruf akkumulierte Humankapital. Die dritte Variable ist binär und nimmt den Wert eins an, wenn der Befragte seinen Beruf gewechselt hat, bzw. null wenn es nicht der Fall ist.

Bei dem Ansatz dieser Studie werden einzelne Tätigkeiten im Gegensatz zu Autor et al. (2003) nicht in größeren Kategorien eingeordnet, sondern als voneinander unabhängige Dimensionen des Tätigkeitenraumes aufgefasst.

In der empirischen Modellierung wird eine modifizierte Mincer'sche Lohnregression geschätzt, um den Zusammenhang zwischen dem individuellen Lohn und einem Berufswechsel zu untersuchen. Das Modell wird getrennt für die Jahre 1991 und 1998 geschätzt, wobei 1991 die kurzfristige und 1998 die langfristige Auswirkung eines Berufswechsels darstellt. Das Modell differenziert außerdem zwischen westund ostdeutschen Beschäftigten. Eine nähere Beschreibung des Schätzkonzepts ist in Fedorets (2011a) zu finden.

Die Ergebnisse für Westdeutschland im Jahr 1991 zeigen, dass diejenigen Beschäftigten, die in ihrem Ausbildungsberuf verblieben sind und die Änderungen der in diesem Beruf ausgeübten Tätigkeiten gemeistert haben, einen Lohnaufschlag bekommen. Dieser empirische Befund steht in Einklang mit der Humankapitaltheorie und weist auf eine Entlohnung des unmittelbar am Arbeitsplatz akkumulierten Humankapitals und die erfolgreiche Anpassung des Arbeitnehmers an die sich ändernden Arbeitsanforderungen hin. Auch im Fall eines Berufswechsels spielt die inhaltliche Entfernung des Wechsels eine signifikante Rolle. Generell gilt, dass Wechsel in inhaltlich weit entfernte Berufe mit Lohnabschlägen verbunden sind. Dies kann als Humankapitalverlust bei einem Berufswechsel interpretiert werden, wobei bei einem Wechsel zwischen ähnlichen Berufen weniger Humankapital verloren geht, als bei inhaltlich distanzierten. Die Transferierbarkeit des Humankapitals hängt positiv mit der Ähnlichkeit zwischen den Ausbildungs- und Erwerbsberufen zusammen, weil inhaltlich ähnliche Berufe auch ähnliche Kenntnisse und Fertigkeiten erfordern. Obwohl ein Teil des Humankapitals verloren geht, erlebt ein westdeutscher Beschäftigter bei einem Berufswechsel im Durchschnitt eine Lohnsteigerung von rund 5 Prozent. Die Steigerung des Wertes des Arbeitnehmer-Karriere Matches (gemessen durch eine binäre Variable, die den Wert eins bei einem getätigten Berufswechsel annimmt) weist darauf hin, dass die meisten Berufswechsel als strategisches Instrument zur Karriereplanung eingesetzt werden, und dass sich die individuelle Produktivität der Beschäftigten im neuen Beruf besser entfaltet. Ähnliche Tendenzen in Bezug auf den $\mathrm{Zu}$ sammenhang zwischen Berufswechsel und Lohn sind auch im Jahr 1998 zu beobachten.

Die empirischen Ergebnisse für Ostdeutschland im Jahr 1991 erfordern eine andere Erklärung. Demnach dürften die Ergebnisse in erster Linie durch den Transformationsprozeß von Plan- zu Marktwirtschaft zu erklären sein. An dieser Stelle ist auch auf die in diesem Heft vorhandene Studie von Orlowski und Riphahn zu verweisen, die ähnliche Grundtendenzen in Bezug auf Humankapitalerträge in Ostdeutschland nach der Wiedervereinigung konstatiert. Grundsätzlich ist nach unseren empirischen Ergebnissen folgendes auf dem ostdeutschen Arbeitsmarkt im Jahr 1991 zu beobachten. Die ostdeutschen Befragten bekommen keinen Lohnaufschlag für die Akkumulation des Humankapitals an einem Arbeitsplatz über die Zeit. Außerdem werden Wechsel in inhaltlich nahe Berufe nicht zusätzlich entlohnt. Diese Befunde weisen darauf hin, dass die meisten Berufswechsel in Ostdeutschland nicht als strategischer Schritt der eigenen Karriere eingesetzt werden konnten. Die Berufswechsel dürften vielmehr auf die mit der Transformation des ostdeutschen Wirtschaftssystems verbundene Veränderung der Berufsstruktur zurückzuführen sein. Obwohl der ostdeutsche Transformationsprozeß als Anlass des Berufswechsels im Jahr 1998 an Wichtigkeit verloren haben dürfte, ist eine weitere Untersuchung dieses Phänomens notwendig. Die wichtigsten Fragen dabei sind, wie negativ und nachhaltig die Auswirkungen der Welle gezwungener Berufswechsel im Durchschnitt waren, die durch die Wiedervereinigung ausgelöst wurden.

Die Analyse in Fedorets (2011b) beschäftigt sich mit diesen Fragen. Dafür wird ein ähnliches Forschungsdesign wie in Fedorets (2011a) verwendet, d.h. eine Schätzung der Lohnregressionen unter Berücksichtigung von Berufswechseln. Der Fokus ist hier allerdings nicht mehr auf die Unterscheidung zwischen Theorien gerichtet, sondern auf die Persistenz der Effekte. Die Schätzung wurde mit Hilfe der Methode der kleinsten Quadrate durchgeführt, wobei die kurz- und langfristige Korrelation zwischen Lohn und Berufswechsel als negativ geschätzt wurde. Im Jahr 1991 beträgt die Korrelation zwischen einem Berufswechsel und logarithmiertem Lohn circa -0,1. D.h. der Berufswechsel ist mit im Durchschnitt 10 Prozent niedrigerem Lohn verbunden. Im Jahr 1998 fällt dieser Wert bis auf -0,04 bzw. 4 Prozent und wird außerdem insignifikant. Allerdings lassen diese Werte mögliche Selektionseffekte außer Acht. Es 
ist plausibel anzunehmen, dass Berufswechsler im Durchschnitt besser beobachtbare und nicht-beobachtbare Qualitäten für den Arbeitsmarkt aufweisen, als Nichtwechsler. Diese Annahme wird durch die empirische Literatur über freiwillige Arbeitsplatz- und Berufswechsel bestätigt. Die grundlegende Idee dabei ist, dass vor allem diejenigen Beschäftigten nach einem Berufswechsel streben, die darin eine höhere Stufe auf der Karriereleiter sehen. Im Durchschnitt erwarten die Berufswechsler, dass sich ihre Arbeitsqualitäten im neuen Beruf besser entfalten können.

Um diesen Selektionseffekt zu kontrollieren und den Kausaleffekt eines Berufswechsels auf den Lohn zu messen, wird in der Schätzung von Fedorets (2011b) eine Instrumentvariable benutzt. Als valides Instrument für die Schätzung erscheint der Ausbildungsberuf, der noch in der DDR erlernt wurde. Die Ergebnisse unter der Verwendung des Instruments zeigen ebenfalls, dass der Kausaleffekt eines Berufswechsels für diejenigen, die in Ostdeutschland geblieben sind, im Jahr 1991 mehr als 35 Prozent beträgt. Dieser negative Effekt ist über die Jahre nachhaltig. So wird im Jahr 1998 eine signifikante negative Wirkung von über 20 Prozent von Berufswechsel auf Löhne festgestellt. Diese Ergebnisse machen deutlich, dass die grundlegenden Änderungen der Berufsstruktur in Ostdeutschland nach der Wiedervereinigung eine signifikante und persistente Wirkung auf den Berufswechsel und Löhne haben, wenn man für die positive Selektivität der Berufswechsler kontrolliert.

\section{Fazit und Ausblick}

Insgesamt erhärten die Ergebnisse der empirischen Studien (Fedorets 2011a, 2011b) die These, dass den Absolventen des deutschen dualen Ausbildungssystems ein hohes Maß an Berufsmobilität zukommt. Humankapital, das diese Absolventen während ihrer Ausbildung akkumuliert haben, ist den Ergebnissen zufolge zwischen unterschiedlichen Berufen transferierbar und kann mit steigenden Löhnen einhergehen. Diese berufsübergreifende Verwertbarkeit der Ausbildungsqualifikationen spricht dafür, dass dieses System Lerninhalte bietet, die den Anforderungen eines modernen Wirtschaftssystems gerecht werden. Dieses positive Ergebnis sollte jedoch nicht über die Notwendigkeit zur Modernisierung der dualen Ausbildung hinwegtäuschen, die durch den technologischen Fortschritt erforderlich wird.

Die Analyse der einschlägigen Literatur zeigt, dass der einzigartige institutionelle Aufbau des deutschen dualen Ausbildungssystems ein effizientes Zusammenspiel von marktbasierten und staatlich regulierten Elementen darstellt. Die Akkumulierung von Kenntnissen und Fertigkeiten unterliegt staatlicher Qualitätssicherung und Standardisierung, wobei die unternehmensbasierte Lehre die nötige Praxisnähe des Ausbildungsprogramms gewährleistet. Allerdings darf man nicht vergessen, dass Produktionsprozesse in der modernen Wirtschaft hohen technologischen Anforderungen unterliegen und somit auch in einem engen Zusammenhang zu Bildung und Wissenschaft stehen.

Es bleibt festzuhalten, dass insbesondere die Verwendung des tätigkeitsbasierten Ansatzes neue Perspektiven bei der Analyse der Transferierbarkeit des Humankapitals und möglicher Modernisierungspotenziale des dualen Ausbildungssystems eröffnet. Der im Folgenden vorgestellte Ausblick auf einen möglichen Weg zu Flexibilisierung des dualen Ausbildungssystems basiert auf den theoretischen Überlegungen des tätigkeitsbasierten Ansatzes und ist aus der eigenen empirischen Analyse nicht direkt ableitbar. Die empirischen Analysen der beiden Studien von Fedorets als auch von anderen Autoren zeigen, wie sich die Flexibilität des erlernten Humankapitals erhöht, wenn man sich von der Ebene der Berufe auf die Ebene einzelner Tätigkeiten begibt. Die Verwendung des tätigkeitsbasierten Ansatzes legt den Gedanken nahe, dass die Flexibilität der Auszubildenden verbessert werden könnte, wenn die klassischen Lehrinhalte durch Zusatzmodule zum Erlernen bestimmter Tätigkeiten ergänzt würden. Eine flankierende Modulstruktur - wie etwa die Wahl des Haupt- und Nebenfaches im Studium - würde es den Auszubildenden ermöglichen, sich ein fachübergreifendes Tätigkeitsprofil anzueignen. Damit wäre der Absolvent nicht nur auf Ausbildungsberufe vorbereitet, sondern auch auf Tätigkeiten jenseits seiner gewählten Berufsbilder.

\section{Executive summary}

This article provides an overview of the results from two empirical studies (Fedorets 2011a, 2011b) devoted to occupational mobility and its correlation with individual wages of the former apprentices of the German Dual Apprenticeship System. This apprenticeship system is one of the most important educational institutions in Germany, the roots of which date back to the medieval guild system. Nowadays, nearly $70 \%$ of young adults enter the labor market through this system. Occupational mobility is inherent to an economy exposed to technological progress. Some professions become obsolete and new professions emerge due to technological changes, while the contents of occupations also change over time. The standard human capital theory predicts wage losses following an occupational change, since part of the human capital accumulated in the old occupation cannot be used in the new one. The search and match theory explains that an occupational change can lead to a wage increase when the match between the worker and his or her profession is enhanced by such a change. Therefore, according to these two theories, the association between an occupational change and subsequent wage alteration is based on three important components: 
1. Usage of accumulated human capital stock and its transferability between different occupations in the case of an occupational change;

2. Changes in the optimality of the match between the employee and his or her profession;

3. Adjustment of individual qualification to the permanently changing content of the occupation.

In the empirical framework of Fedorets (2011a), several variables are applied to disentangle the correlation between each component and wages. First of all, the transferability of human capital is measured by employing distance measures between different occupations with regard to their contents. Secondly, an increase or a decrease in the optimality of the match between the employee and his or her profession is measured using a binary variable that takes a value of 1 in the case of an occupational change and 0 otherwise. Thirdly, the adjustability of qualifications is captured by measuring the similarity of contents for the same occupation at different time points. Based on these variables, the three components that determine the association between occupational changes and subsequent wages can be used in the estimation of a wage regression. The estimation was made using a sample of male German employees with apprenticeship training as their highest educational level taken from the Qualification and Career Survey for the years 1991 and 1998. The main advantages of this survey are that it has a special focus on the occupational careers of the respondents and that it contains information on the apprenticeship qualifications, as well as detailed information on the tasks performed in respondents' position of employment at the time of the survey. OLS estimation shows that for West German employees, human capital is more transferable between occupations with similar task content. An occupational change is associated with higher wages, which implies that, on average, the quality of the employee-profession match improves. Adjustment of qualifications to the permanently changing content of the occupation is also rewarded. The estimated coefficients for East Germany, on average, imply more negative consequences of an occupational change compared to the West German subsample. During the period 1991-1998, the results were mainly driven by the process of transformation from a planned to a market economy.

The magnitude and persistence of the negative wage effects of an occupational change in East Germany after reunification were analyzed in Fedorets (2011b). The OLS regression estimates the correlation between occupational changes and wages as being significantly negative in 1991 and negative but insignificant in 1998. However, these numbers do not take into account the positive selectivity of the sample of those who have changed their occupation. In order to control for selectivity, post-reunification changes in economic structure-implying changes in demand for particular occupational groups-were used as basis for an instrument for occupational changes. The IV estimation reveals that the negative wage effect of an occupational change between 1990 and 1991 amounts to about 35\%. Wage losses due to occupational changes between 1990 and 1998, i.e. almost 10 years after reunification, amount to nearly $20 \%$. It could thus be shown that fundamental changes in the occupational structure in East Germany have caused persistent wage losses for medium-skilled workers.

On the whole, the empirical findings of both studies support the hypothesis that human capital accumulated during apprenticeships is transferable between occupations. The magnitude, significance and persistence of the effect of occupational changes on wages depend on the similarity between the occupations before and after the occupational change, as well as on the underlying economic conditions that may influence the individual decision to change professions.

\section{Literatur}

Acemoglu, D., Pischke, J.-S.: Why do firms train? Theory and evidence. Q. J. Econ. 113(1), 79-119 (1998)

Acemoglu, D., Pischke, J.-S.: The structure of wages and investment in general training. J. Polit. Econ. 107(3), 539-572 (1999)

Autor, D.H., Levy, F., Murnane, R.J.: The skill content of recent technological change: an empirical exploration. Q. J. Econ. 118(4), 1279-1333 (2003)

Ehrenberg, R., Smith, R.: Modern Labor Economics. Theory and Public Policy, 9 Aufl. Pearson, Upper Saddle River (2006)

Fedorets, A.: Disentangling the complex impact of occupational mobility on wages using the task-based approach (2011a, work in process)

Fedorets, A.: Transitional changes in the occupational structure and their impact on individual wages (2011b, work in process)

Fitzenberger, B., Kunze, A.: Vocational training and gender: wages and occupational mobility among young workers. ZEW Discussion Paper 05-66, S. 1-41 (2005)

Fitzenberger, B., Spitz, A.: Die Anatomie des Berufswechsels: Eine empirische Bestandsaufnahme auf Basis der BiBB/IAB Daten 1998/1999. ZEW Discussion Paper 04/02, S. 1-41 (2004)

Franz, W.: Arbeitsmarkökonomik, 6 Aufl. Springer, Heidelberg (2006)

Franz, W., Soskice, D.: The German Apprenticeship System. In: Buttler, F., et al. (Hrsg.) Institutional Frameworks and Labor Market Performance: Comparative Views on the U.S. and German Economies. Routledge, London (1995)

Franz, W., Zimmermann, V.: Mobilität nach der beruflichen Erstausbildung: Eine empirische Studie für Westdeutschland. ZEW Discussion Paper 97-21, S. 1-34 (1999)

Gathmann, C., Schoenberg, U.: How general is human capital? A taskbased approach. J. Labor Econ. 28(1), 1-49 (2009)

Harhoff, D., Kane, T.: Financing apprenticeship training: evidence from Germany. NBER Working Paper Series 4557, 1-46 (1993)

Harhoff, D., Kane, T.: Is the German apprenticeship system a panacea to the U.S. labor market? J. Popul. Econ. 10, 171-196 (1997)

Herget, H., Schöngen, K., Westhoff, G., Menk, A., Schiemann, M., Sprang, B., Walz, U.: Berufsausbildung abgeschlossen - was dann? Berichte zur beruflichen Bildung 85, 1-156 (1985)

Hofbauer, H., Nagel, E.: Mobilität nach Abschluss der betrieblichen Berufsausbildung. Mitt. Arb.- Berufsforsch. 20, 45-73 (1987) 
Johnson, W.R.: A theory of job shopping. Q. J. Econ. 92(2), 261-277 (1978)

Korpi, T., Mertens, A.: Training systems and labor mobility. In: SFB 373 (2003) 19, 1-35

Münch, J.: Vocational education and training in the Federal Republic of Germany. CEDEFOP (1995)

Neal, D.: The complexity of job mobility among young men. J. Labor Econ. 17(2), 237-261 (1999)

Parnes, H.S.: Research on Labor Mobility. An Appraisal of Research Findings in the United States. Social Science Research Council, New York (1954)

Sharpe, A., Gibson, J.: The apprenticeship system in Canada: trends and issues. Cent. Study Living Stand. 4, 2-98 (2005)

Spitz-Oener, A.: Technical change, job tasks, and rising educational demands: looking outside the wage structure. J. Labor Econ. 24(2), 235-270 (2006)

Streek, W., Hilbert, J., van Kevelaer, K.-H., Maier, F., Weber, H.: The role of the social partners in vocational training and further training in the Federal Republic of Germany. CEDEFOP (1987)

Topel, R., Ward, M.: Job mobility and the careers of young men. Q. J. Econ. 107(2), 439-479 (1992)

Von Wachter, T., Bender, S.: In the right place at the wrong timerole of firms and luck of the young worker's careers. Am. Econ. Rev. 96(5), 1679-1705 (2006)

Werwatz, A.: Mobility after apprenticeship. How effective is the German apprenticeship system? Sonderforschungsbereich $373 \mathbf{7 5}, 1-$ 32 (1997)

Winkelmann, R.: Employment prospects and skill acquisition of apprenticeship-trained workers in Germany. Ind. Labor Relat. Rev. 49(4), 658-672 (1996a)

Winkelmann, R.: Training, earnings and mobility in Germany. Konjunkturpolitik 42(2), 159-170 (1996b)
M.Sc. Alexandra Fedorets Studium der Volkswirtschaftslehre an der Lomonossow-Universität, Moskau. 2007 Abschluss als Master of Science im Rahmen des „Master's Program in Economics and Management Science“ der Humboldt-Universität zu Berlin. Seit 2007 Doktorandin im „Berlin Doctoral Program in Economics and Management Science“. 2008-2011 Mitarbeiterin am Lehrstuhl für Angewandte Mikroökonomik der Wirtschaftswissenschaftlichen Fakultät der Humboldt-Universität zu Berlin.

Forschungsfelder: Arbeitsmarktökonomie, insbesondere berufliche Mobilität und damit verbundene Lohnentwicklungen.

Prof. Dr. Alexandra Spitz-Oener Studium der Volkswirtschaftslehre an der Universität Mannheim. 2000 Abschluss als Diplom-Volkswirtin. 2004 Promotion zum Dr. rer. pol. an der Universität Mannheim. 2005 Marie-Curie „Post-Doctoral Position“ am „Centre du Recherche en Économie et Statistique (CREST)“, Paris. Seit 2006 Professorin für Volkswirtschaftslehre an der Humboldt-Universität zu Berlin (bis Anfang 2010 als Juniorprofessorin). Seit 2008 Research Fellow am Institut für Arbeitsmarkt- und Berufsforschung der Bundesagentur für Arbeit. Seit 2009 Projektleiterin im DFG-Sonderforschungsbereich 649 „Ökonomisches Risiko“, seit 2010 Projektleiterin im Forschungsprogramm „Migration in Europe“, 2008-2011 DFG-Projekt „Flexibilisierungspotentiale bei heterogenen Arbeitsmärkten“. Seit 2007 IZA Research Fellow, seit 2008 Mitglied des Bevölkerungsökonomischen Ausschusses des Vereins für Socialpolitik, seit 2010 gewähltes Mitglied des Exekutivkomitees der European Association of Labor Economists (EALE).

Forschungsfelder: angewandte Arbeitsmarktökonomie, insbesondere Wirkungen von technologischen Veränderungen auf die Arbeitsnachfrage und Effekt von Produktmarkt- und Arbeitsmarktregulierung auf Löhne und Beschäftigung. 